\title{
Nuclear factor I revealed as family of promoter binding transcription activators
}

\author{
Milos Pjanic ${ }^{1{ }^{*}+}$, Petar Pjanic ${ }^{2+}$, Christoph Schmid ${ }^{3,4,5,6}$, Giovanna Ambrosini ${ }^{3}$, Armelle Gaussin ${ }^{1}$, Genta Plasari ${ }^{1}$, \\ Christian Mazza ${ }^{7}$, Philipp Bucher ${ }^{3}$ and Nicolas Mermod ${ }^{1}$
}

\begin{abstract}
Background: Multiplex experimental assays coupled to computational predictions are being increasingly employed for the simultaneous analysis of many specimens at the genome scale, which quickly generates very large amounts of data. However, inferring valuable biological information from the comparisons of very large genomic datasets still represents an enormous challenge.
\end{abstract}

Results: As a study model, we chose the NFI/CTF family of mammalian transcription factors and we compared the results obtained from a genome-wide study of its binding sites with chromatin structure assays, gene expression microarray data, and in silico binding site predictions. We found that NFI/CTF family members preferentially bind their DNA target sites when they are located around transcription start sites when compared to control datasets generated from the random subsampling of the complete set of NFI binding sites. NFI proteins preferably associate with the upstream regions of genes that are highly expressed and that are enriched in active chromatin modifications such as H3K4me3 and H3K36me3. We postulate that this is a causal association and that NFI proteins mainly act as activators of transcription. This was documented for one member of the family (NFI-C), which revealed as a more potent gene activator than repressor in global gene expression analysis. Interestingly, we also discovered the association of NFI with the tri-methylation of lysine 9 of histone $\mathrm{H} 3$, a chromatin marker previously associated with the protection against silencing of telomeric genes by NFI.

Conclusion: Taken together, we illustrate approaches that can be taken to analyze large genomic data, and provide evidence that NFI family members may act in conjunction with specific chromatin modifications to activate gene expression.

\section{Background}

High-throughput assays are being widely employed in various fields of biology. For example in genomics, DNA microarrays are used to simultaneously measure the expression levels of nearly all genes of a genome [1,2]. Recently, a new high-throughput method has been developed for a whole genome mapping of protein-DNA interactions that is based on the chromatin immunoprecipitation and next generation sequencing technology (method termed chromatin immunoprecipitation sequencing or ChIP-Seq) [3-8]. These two high-

\footnotetext{
* Correspondence: pjanic.milos@gmail.com

+ Contributed equally

'Institute of Biotechnology, University of Lausanne, and Center for Biotechnology of the University of Lausanne and École Polytechnique

Fédérale de Lausanne, CH-1015 Lausanne, Switzerland Full list of author information is available at the end of the article
}

throughput methods, when combined, are instrumental to study how transcription factors regulate gene expression at a global genomic scale. As the costs of new-generation sequencing and DNA microarrays decrease, such high-throughput assays should be increasingly used. In addition, new software tools are emerging rapidly, allowing faster and easier analyses of large-scale genomic datasets [9-13]. However, extracting the significant and biologically relevant information from such massive datasets still represents a great challenge. In purely experimental studies, the use of negative controls such as the blank or mock conditions is absolutely necessary. However, genome-wide computer analyses may lack an adequate negative control. In such case, a randomly selected portion of the total dataset can be used as an in silico negative control, such as for instance a randomly picked sample of all genomic loci. The size of the
C Biomed Central 
control dataset can then be chosen to parallel that of the experimental set, to simplify the statistical analysis $[14,15]$.

As a study group of proteins, we chose NFI/CTF family of mammalian transcription factors. NFI/CTF represents a family of transcription-replication factors comprising polypeptides encoded by four paralogous genes located on different chromosomes in mammals (NFIA, NFIB, NFIC, NFIX) $[16,17]$. NFI family of proteins displays the unusual property of regulating not only the initiation of transcription but also of mediating DNA replication [18]. NFI recognition sequence were found in the promoter sequences of many cellular genes [19], where they may act as activator or repressor of transcription [20-25]. Recently, it has been proposed that NFI may be involved in a long range regulation of gene expression, through the formation of chromatin barrier and by blocking the propagation of a heterochromatic structure [26,27]. NFI binds as a dimer, and its preferred binding sequence is a palindrome composed of two half sites TTGGCANNNTGCCAA. A position weight matrix for the NFI/CTF was established using a collection of over 10,000 SELEX-SAGE selected sites, allowing the prediction of its binding affinity to any genomic sequence [28]. However, since this prediction matrix is based on NFI binding specificity in vitro, the specificity of this family of proteins may be different from that observed in the cell, where interactions with other transcription factors may take place and DNA accessibility may be restrained by chromatin. Here we assessed the in vivo binding preferences of NFI/CTF, its global functional properties regarding the regulation of gene expression and the relationship of NFI binding sites with different histone methylation markers typical of either an open or closed chromatin structure.

\section{Results}

\section{NFI preferentially binds upstream of transcription} initiation sites in mouse genome

Statistical analysis in genomics often relies on the subsampling of datasets, which requires random sampling algorithms. We devised a random sampling algorithm that can be conveniently applied to large genomic datasets. The random sampling algorithm $\mathrm{C}++$ source code is available as a text file online (Additional file 1). Each randomly generated number is used to extract an entry line from the main dataset to generate a subset of the desired size. Simulation experiments indicated that subsampling can be applied to sets of normally distributed values without loosing the statistical robustness of the comparisons, provided that relatively large subsets of data are retained (e.g. equal or greater than 100 individual values; see Additional file 2).
We first used this random sampling method to compare data from a ChIP-Seq experiment performed on primary mouse embryonic fibroblasts for the NFI transcription factor family relative to the in silico predictions of its binding sites. The mouse genome (NCBI build 37 or $\mathrm{mm} 9$ ) was found to contain a set of 61,492 NFI predicted sites that were defined using a previously established position weight matrix $[19,28]$. The predicted sites were defined with a matrix score threshold $>85$, which corresponds to a medium in vitro binding affinity in the range of scores that extends from a minimum of -108 to a maximum of 100 . Within this set, 2,852 predicted sites overlapped DNA sequences covering the RefSeq annotated transcription start sites (TSS) and 5 $\mathrm{kb}$ of upstream sequences.

Actual in vivo binding site occupancy was estimated using the average ChIP-Seq tag count covering each subset of NFI predicted sites. When tag counts occurring at TSS-proximal binding sites were compared to those that do not occur near known TSS, a higher tag counts was noted at the predicted sites, but also in regions extending several $\mathrm{kb}$ away from the known binding sites (Figure 1A). Since the DNA fragments used in this assay did not cover a size range extending over several $\mathrm{kb}$, we reasoned that this might either result from a surprisingly frequent association of the protein to sequences near binding sites, or, alternatively, that it may stem from the smaller size of the dataset comprising the TSS-proximal 2,852 binding sites relative to the non-TSS datasets of 58,640 sequences, resulting in a relatively noisier distribution at non-bound sequences, or from some other artefactual effects associated to the method.

To assess the latter possibility further, we generated control datasets of the same size as the experimental set by randomly selecting 2,852 sequences from the 61,492 predicted sites. Comparison of distinct randomly selected data subsets indicated comparable tag counts at predicted binding sites, and similar signal-to-noise ratio. Thus, the sampling method provided a reliable estimation of the binding site occupancy, since several random groups of predicted sites did not differ markedly in their protein occupancy (Figure 1B). As before, the dataset corresponding to TSS-proximal sites showed a more prominent tag count around the predicted sites. However, comparable tag counts were observed within 500 to $5000 \mathrm{bp}$ windows around the predicted binding sites, when comparing the experimental profiles to those of control datasets of the same size. Thus, we conclude that the group of TSS-proximal predicted sites displayed higher protein occupancy at predicted sites when compared to the randomly selected binding sites, but that it was not the overall promoter region that was more frequently bound by the protein. This suggested that 

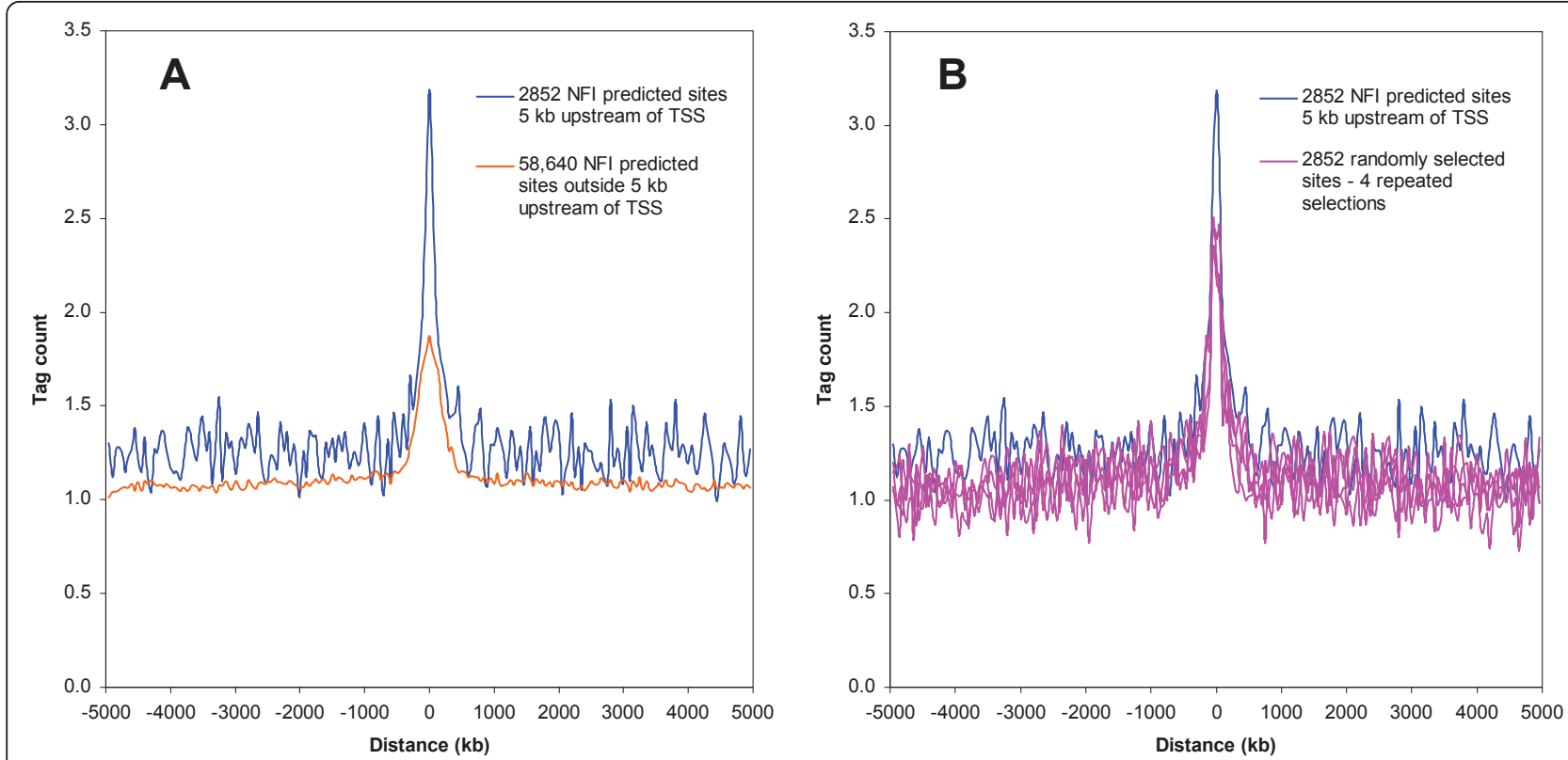

Figure 1 NFI family of DNA binding proteins preferentially binds upstream of initiation sites of transcription in mouse genome. A set of $61,492 \mathrm{NFI}$ predicted sites was defined in the mouse genome, NCBI build 37 or mm9. Sites were defined with a previously established position weight matrix (sequence score threshold $>85$, out of maximum 100) [28]. Within this set, 2,852 predicted sites overlapped regions $5 \mathrm{~kb}$ upstream from RefSeq annotated transcriptional start sites (TSS). As a negative control for this experiment, a complementary dataset of 58,640 sites was selected (panel A) or the same number of NFI predicted sites $(2,852)$ were randomly selected from the initial set of 61,492 predicted sites (panel B). Four independently performed random selection are shown. Average NFI ChIP-Seq tag counts were calculated in windows of 50 bp for a region of $5 \mathrm{~kb}$ up- and down-stream of the selected NFI predicted sites. Tag counts were normalized globally, as a fold increase over the genome average tag count in a window of $50 \mathrm{bp}$. Obtained data points were connected to form a continuous line.

binding sites within genomic loci upstream of core promoters may bind NFI with higher apparent affinity.

\section{NFI-bound genes show higher expression levels}

Next, we assessed whether genes that bind NFI may have some common features regarding their gene expression levels. We selected 39,807 in vivo NFI sites genome-wide from the ChIP-Seq data, which corresponds to the genomic loci obtained from the collection of precipitated DNA fragments. Out of this number, 3,120 in vivo sites were located within $5 \mathrm{~kb}$ upstream regions of RefSeq annotated genes. As multiple in vivo NFI sites may occur within the $5 \mathrm{~kb}$ upstream regions, a total of 2881 RefSeq-annotated genes were identified to contain one or more in vivo NFI sites. Control groups were generated to consist of 2,881 genes randomly selected from the total database of RefSeq genes. The expression levels of these genes was assessed using microarray profiling data of mRNAs obtained from murine embryo fibroblasts [29]. Again, randomly selected groups of genes did not differ significantly in their overall expression levels or in their distribution profiles, with the most prominent peak corresponding to lowly expressed genes (Figure 2A-C). However, the transcriptional levels of the group of NFI-containing genes were distinctly higher than those of the control subgroups (Figure 2D), with a median expression value of 6.34 for NFI-bound genes versus 4.93, 4.97 and 4.82 for the randomly selected groups of genes (two tailed t-test: $\mathrm{p}=$ $8.94 \times 10^{-61}, 1.15 \times 10^{-57}, 1.35 \times 10^{-65}$, respectively). Thus, we conclude that NFI preferentially occupies promoters or upstream regulatory regions of genes that exhibit high expression levels in the cell.

\section{NFI binding correlates with specific histone methylation patterns}

To test whether the preferential binding of NFI to upstream regions of expressed genes may be associated with specific chromatin modifications, we used ChIPSeq data for 4 different histone $\mathrm{H} 3$ methylations, as previously obtained from mouse embryonic fibroblasts [4]. We created average ChIP-Seq tag profiles surrounding the TSS for each of these modifications. The group of 2,881 NFI bound genes showed higher levels of H3K4me3 and H3K36me3, which are markers of active promoters and transcribed regions, respectively, when compared to the three groups of 2,881 randomly chosen genes (Figure 3A, B). Interestingly, NFI binding at TSS did not correlate with a modification often associated with non-expressed or bivalent chromatin, H3K27me3, 


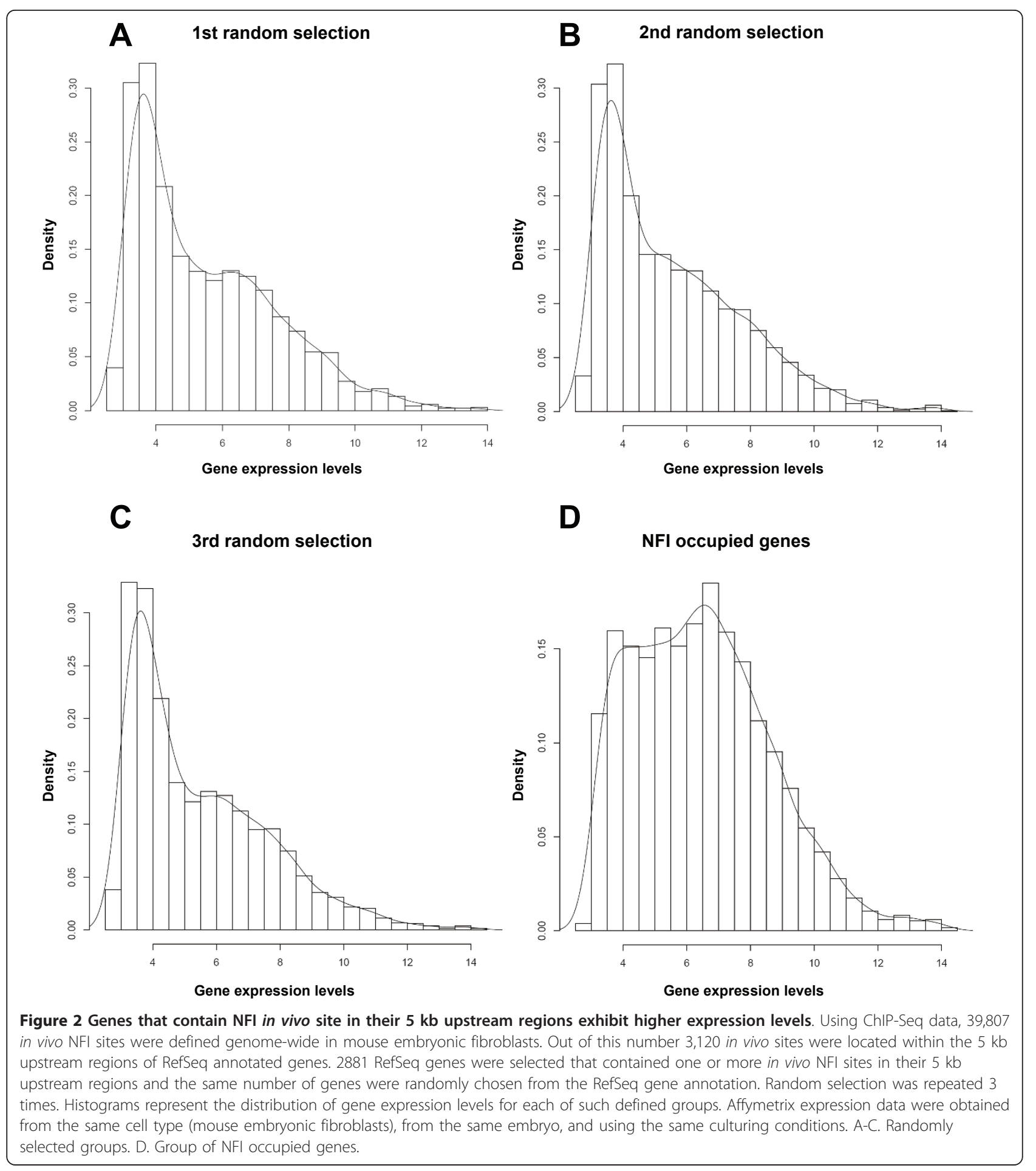

when compared with the profiles of the control gene subgroups (Figure 3C), indicating that NFI binding does not occur at promoter regions that are generally enriched in all histone modifications. Interestingly, we found H3K9me3, a modification recently associated with NFI chromatin-domain boundary activity at telomeres
[26], to be also enriched surrounding the TSS of the group of NFI-bound genes (Figure 3D).

NFI-C most often acts as an activator of gene expression The preferential association of NFI with expressed genes prompted us to test whether NFI family members may 


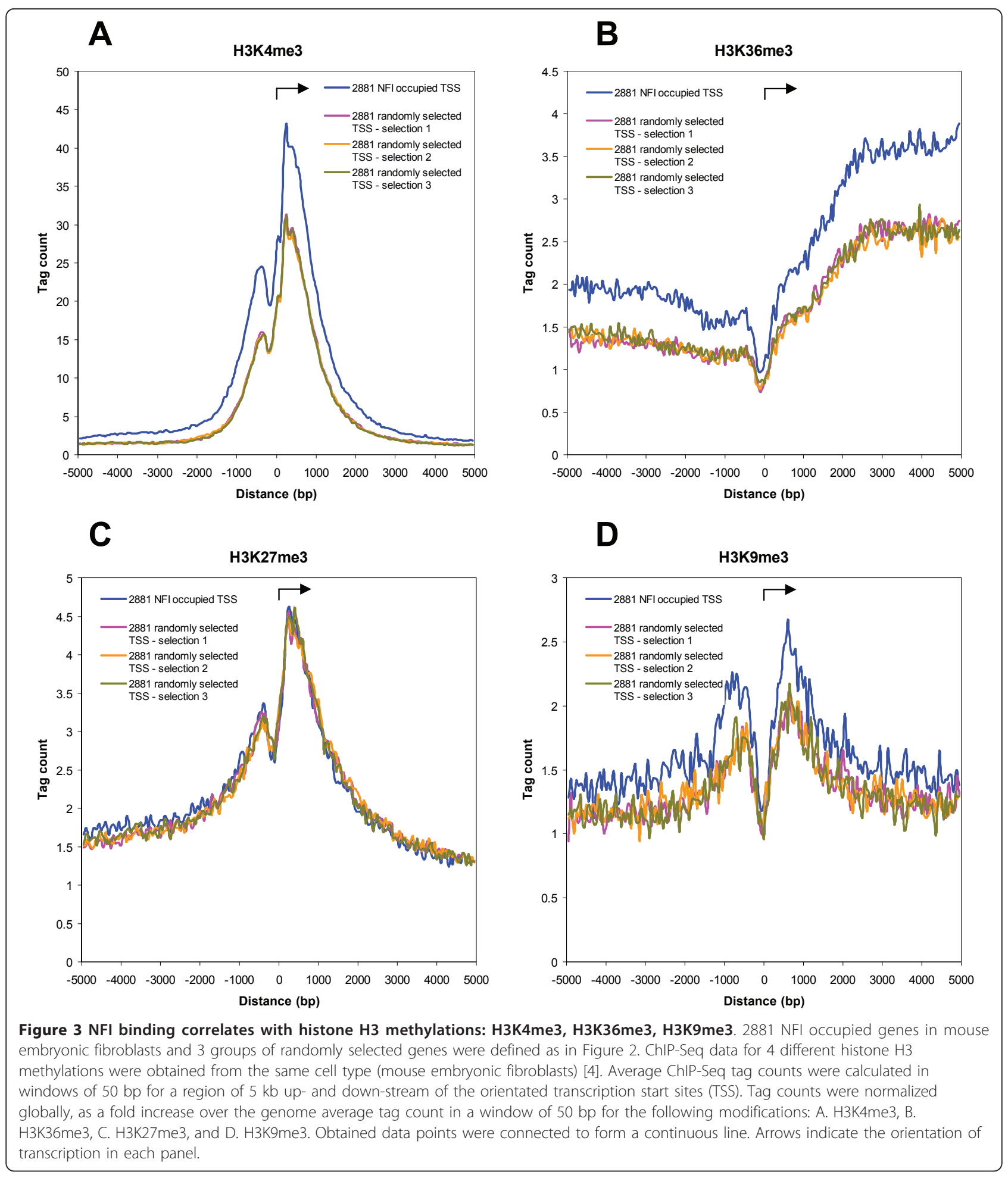

have the potential to activate gene expression, or whether their binding is rather the consequence of histone modifications and chromatin accessibility at promoters. For this purpose, we used gene expression microarray data from mouse embryonic fibroblasts in which one of the NFI factors (termed NFI-C) was knocked out by insertional mutagenesis [30]. We focused on differences in expression levels when comparing wild-type (WT) and knock-out cells (KO), and defined a set of 1000 genes which expression was most 
decreased in the absence of NFI-C (i.e. genes potentially directly activated by this factor). We also defined the set of 1000 genes whose expression is most increased in the absence of NFI-C (i.e. genes potentially directly repressed by NFI-C). We also randomly sampled 1000 genes from all RefSeq genes as control sets. Differences in the microarray signals of WT and $\mathrm{KO}$ cells $(\Delta \mathrm{E}=$ $\left.E_{w t}-E_{k o}\right)$ were determined for each gene within defined groups and plotted as an absolute value. The group of genes activated by NFI-C showed the highest median $\Delta \mathrm{E}$ value, implicating this factor as a potent gene activator (Figure 4A). NFI-C suppressed genes also appeared to be significantly regulated as compared to the control groups. However, NFI-C suppressed genes displayed moderate $\Delta \mathrm{E}$ values, between those of the control groups and of the group of NFI-C activated genes, indicating that NFI-C is globally a less potent inhibitor of gene expression. When considering the expression levels in WT cells $\left(E_{w t}\right)$, the group of NFI-C suppressed genes had levels of expression that did not differ significantly from the control subgroups (median expression value: 5.16 for down-regulated genes versus $4.81,4.67$ and 4.78 for the control gene groups, two tailed t-test: $p=0.97$, 0.57 , and 0.50 , respectively), again indicating that NFI-C acts as a moderate inhibitor of gene expression (Figure 4B). Overall, the group of NFI-C activated genes had significantly higher levels of expression than the control groups (median expression value: 6.18 for up-regulated genes versus $4.81,4.67$ and 4.78 for random gene groups; two tailed t-test: $\mathrm{p}=2.04 \times 10^{-19}, 3.22 \times 10^{-21}$, $1.60 \times 10^{-22}$, respectively). These results suggest that NFI-C member of the NFI family acts globally as an effective gene activator.

\section{Discussion and Conclusions}

In this study, we used a random sampling procedure as a general method to obtain reliable control datasets in the analysis of high-throughput genomic assays. We find that datasets of more than 100 individual values can be used without decreasing the robustness of statistical analysis, and that independently generated random subsets of data have statistically indistinguishable global properties. Thus, subsampling can provide a convenient way to display and compare the noise and signals from experimental and control datasets of the same size.

First, we showed that NFI binds preferentially those predicted sites that are located upstream of the initiation sites of transcription (Figure 1). Several interpretations may be given to the preferential association of NFI to binding sites in the proximity of TSS rather than to other locations of the genome. It is known that NFI occupies the promoters of many genes where it may bind synergistically with some other transcription factors such as hepatocyte nuclear factor 1 alpha, estrogen receptor, Brg- associated factor [31-33]. Thus, the preferred occupancy of TSS proximal sites may at least in part reflect the synergistic association of NFI with other factors.

We also found that NFI occupy promoters or upstream regions of the group of genes that are significantly more expressed than the representative randomly selected control groups. Since correlation does not necessarily imply causal relationship, this observation does not allow the conclusion that NFI-family members actually activate the expression of these genes. For instance, NFI might bind highly expressed genes to suppress in part their expression, but still leaving relatively high transcription levels. However, taken together with previous observations that NFI activates the expression of many genes in higher eukaryotes [20,21,32,34-37], we rather conclude that the observed correlation may originate from a direct up-regulation of gene expression by NFI, at least for a significant proportion of its target genes.

The hypothesis that NFI family members may directly activate genes appears to be true for at least one of the member of the family (i.e. NFI-C), as mRNA profiling analysis performed on wild-type and NFI-C knock-out cells revealed that NFI-C is a more potent gene activator than a repressor. The 1000 genes that are most up-regulated by NFI-C had significantly higher change in their expression levels than the top 1000 down-regulated genes. In addition, up-regulated genes showed significantly higher expression levels than representative control gene samples selected from the total gene population, implicating again that this factor is a potent activator of gene expression. Since the selected in vivo NFI binding sites are located up to $5 \mathrm{~kb}$ from their TSS, which is a relatively large distance, NFI might act as well through some of the types of remote regulation, for instance by the establishment of a chromatin domain boundary that would prevent the propagation of a silencing chromatin structure towards the promoter $[27,38]$.

Histone H3 methylations such as the H3K4me3 and H3K36me3 modifications were found to be enriched around the TSS of NFI-occupied genes when compared with control gene groups. This finding is consistent with the model that NFI acts predominantly as an activator of transcription, since H3K4me3 and H3K36me3, but not H3K27me3, were proposed as markers of active gene transcription $[4,6,39]$. This indicates that NFI binding to the upstream regions may contribute to the recruitment of the specific enzymes for the H3K4me3 and H3K36me3 modifications. A genome-wide correlation of the occurrence of H3K27me3 was also observed around TSS occurring close to NFI-bound sites, however it was indistinguishable to that of the control group of genes. This indicates that this correlation results from an enrichment of H3K27me3 around at least some of the TSS, and that NFI is not involved in the recruitment of enzymes mediating this 


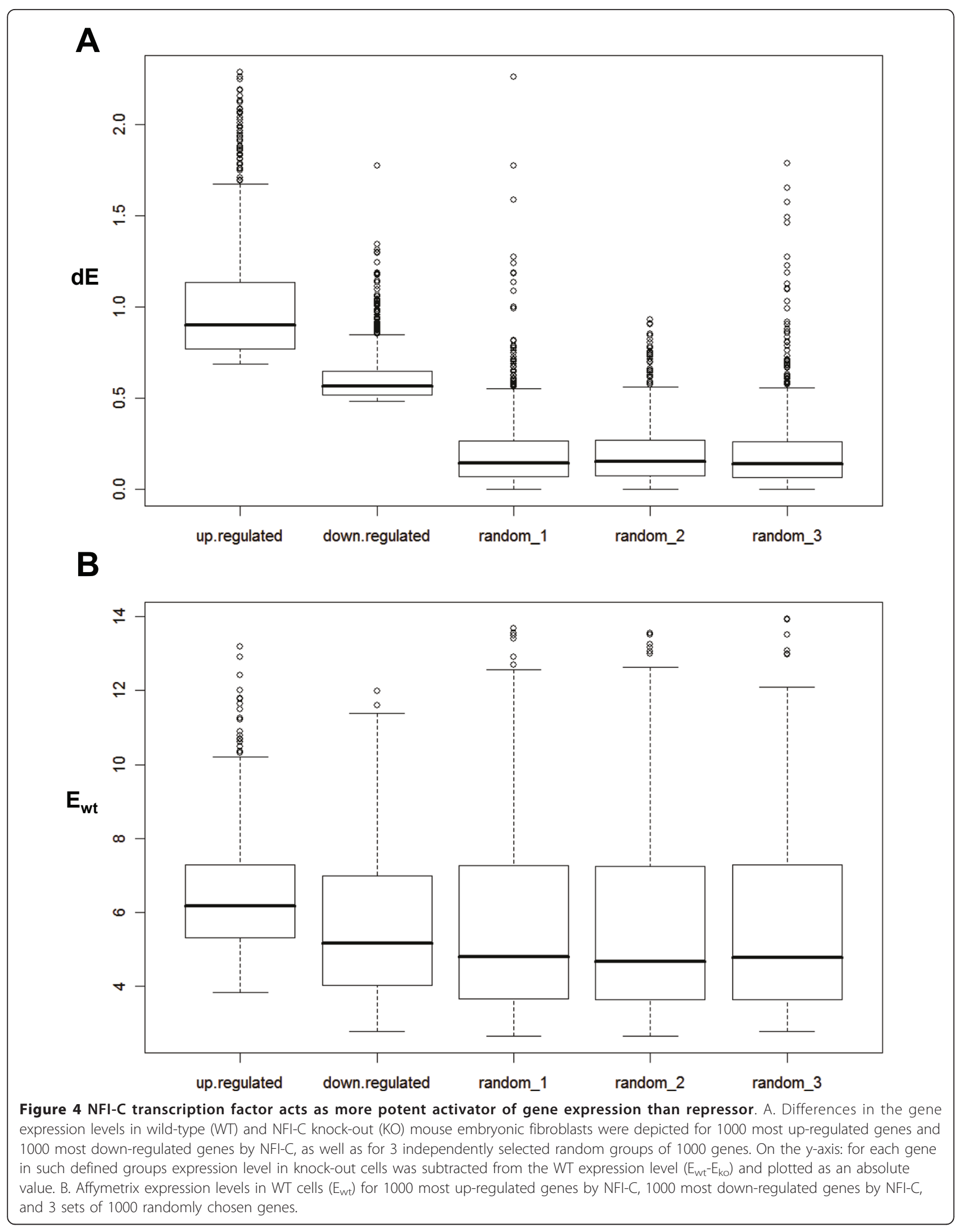


modification. Thus, the enrichment of H3K27me3 modification over the NFI bound genes represents a false positive genome-wide correlation. Interestingly, we also found the H3K9me3 modification to be slightly enriched in the group of NFI bound genes. Although H3K9me3 has been associated with a closed chromatin structure, this suggests that NFI may be involved in the recruitment of enzymes that mediate this modification. Interestingly, this modification was recently associated with a chromatin domain boundary effect at telomeric regions in human cells [26]. In this study, NFI was shown to prevent the propagation of a silencing chromatin structure from the telomere, and the expressed genes protected from telomeric silencing by NFI were shown to have elevated H3K9me3 marks at specific telomeric positions. Thus, we may conclude from these studies that the enrichment in H3K9me3 may be a hallmark of gene expression activation by NFI.

\section{Methods}

\section{Cell culture}

Mouse embryonic fibroblasts (MEF) were extracted from mouse embryos of 14.5 days. Cells from WT (wild-type) and NFI-C knock-out embryos were cultured under the following conditions: $37^{\circ} \mathrm{C}, 5 \% \mathrm{CO}_{2}$, DMEM (GIBCO, 41966), Supplementary 10\% FBS (GIBCO, Fetal Bovine Serum, qualified origin US, 26140-079), 1\% v/v nonessential amino-acids (GIBCO, 11140-035), 1\% v/v L-glutamine (GIBCO, 25030-024).

\section{Chromatin Immuno-precipitation (ChIP)}

Chromatin was extracted from approximately 20,000,000 primary mouse embryonic fibroblasts grown in culture and cross-linked using 11\% formaldehyde. Extracted chromatin was fragmented to the average fragment size of $1000 \mathrm{bp}$ using high-frequency sound sonication on VibraCell-75455 (Bioblock Scientific). ChIP was performed as described before [27] using the commercial antibody against NFI group of proteins (NFI (H300): sc-5567, SantaCruz Biotechnology). Antibody complexes were precipitated using rProtein A Sepharose Fast Flow (Amersham Biosciences).

\section{Illumina/Solexa sequencing}

ChIP DNA was processed using the contents of the ChIP-Seq Sample Prep Kit (Illumina). Size range of templates was selected by loading the entire processed sample on a $2 \%$ agarose gel and excising the gel region of 50-400 bp. PCR amplification of the gel-extracted DNA was performed for 18 cycles using the adapter-specific primers. Each sample was loaded into 3 separate flow cell channels of the Illumina Cluster Station and then subjected to sequencing-by-synthesis on the Illumina Genome Analyzer sequencing system. For each of the samples, sequence reads from independent channels were pooled together.

\section{Data analyses}

Clustering and correlation analyses of mapped reads were performed using ChIP-peak and ChIP-cor tools available on the ChIP-Seq Analysis Server [40,41]. In vivo NFI sites were defined using the ChIP-peak tool and applying the following parameters: window width $300 \mathrm{bp}$, vicinity range $-300 \mathrm{bp}$, peak threshold - 5 tags, count cut-off - 1 tag, repeat filtering-on. Correlation analyses were made using ChIP-cor tool and applying the following parameters: window width - $50 \mathrm{bp}$, count cut-off - 1, normalization - global. Galaxy tools were used to operate on the genomic intervals $[42,43]$.

\section{Random sampling algorithm}

Random sampling algorithm was written in $\mathrm{C}++$ language and compiled in Microsoft Visual Studio as detailed in the Additional file 1 online. The algorithm uses the computer system date and time as a constantly increasing number for seeding the random number generators at each independent run of the random number generator. Each random number so generated was used to select a single entry line from the dataset to be subsampled, with the limitation that a single line of the input file could be selected only once. The source code for random sampling algorithm is available in the additional materials online (Additional file 1).

\section{Datasets repository}

ChIP-Seq data for the wild type and NFI-C knock-out mouse embryonic fibroblasts were deposited at the Gene Expression Omnibus (GEO) repository under the accession number GSE15844. Gene expression microarray data for the wild type and NFI-C knock-out mouse embryonic fibroblasts were taken from the GEO repository under the accession number GSE15871. ChIP-Seq data for histone methylations in mouse embryonic fibroblasts (H3K4me3, H3K9me3, H3K27me3, H3K36me3) were taken from GEO repository under the accession number GSE12241.

\section{Additional material}

Additional file 1: (Source code of the random sampling algorithm, text file).

Additional file 2: (Construction and statistical properties of random sampling method, supplemental figures relating to the construction and statistical properties of the random sampling method).

\section{List of Abbreviations}

NFI: Nuclear Factor I; NFI-C: Nuclear Factor I - C; CTF: CAAT box transcription factors; ChIP: Chromatin immuno-precipitation; ChIP-Seq: Chromatin immuno-precipitation sequencing; MEFs: Mouse embryonic fibroblasts; TSS: Transcriptional start site; TFs: Transcription factors; NFI-C KO: Nuclear Factor I - C knock out; WT: Wild type 


\section{Acknowledgements}

We would like to acknowledge Fasteris, SA, CH-1228 Plan-les-Ouates, Switzerland for providing fast and accurate service of Illumina Genome Analyzer high-throughput DNA sequencing and for processing the data output of obtained sequence tags through ELAND mapping software pipeline. This work was supported by the grants from the University of Lausanne, Switzerland.

\section{Author details}

'Institute of Biotechnology, University of Lausanne, and Center for Biotechnology of the University of Lausanne and École Polytechnique Fédérale de Lausanne, CH-1015 Lausanne, Switzerland. ²Software Examination and Certification Laboratory, Faculty of Mathematics, University of Belgrade, 11000 Belgrade, Serbia. ${ }^{3}$ Swiss Institute for Experimental Cancer Research, École Polytechnique Fédérale de Lausanne, CH-1015 Lausanne, Switzerland. ${ }^{4}$ Swiss Tropical and Public Health Institute, Socinstrasse 57, P.O. Box, CH-4002 Basel, Switzerland. ${ }^{5}$ Universität Basel, Petersplatz 1, CH-4003 Basel, Switzerland. ${ }^{6}$ Swiss Institute of Bioinformatics, EPFL SV ISREC, AAB 009 (Bâtiment AAB), Station 15, CH-1015 Lausanne, Switzerland. 'Department of Mathematics, University of Fribourg, CH-1700 Fribourg, Switzerland.

\section{Authors' contributions}

MP conceived the study, carried out the ChIP-Seq experiments, participated in the data analysis and drafted the manuscript. PP developed the random sampling algorithm and participated in the data analysis. CS, GA and PB developed ChIP-Seq analysis tools and participated in the data analysis. AG, GP made substantial contributions to the data collection and interpretation of data. CM, PB and NM participated in the design of the study, made substantial contributions to the interpretation of data, provided essential data revisions, and helped to draft the manuscript. All authors have read and approved the final manuscript.

\section{Received: 15 August 2009 Accepted: 7 April 2011}

Published: 7 April 2011

\section{References}

1. Planet PJ, DeSalle R, Siddall M, Bael T, Sarkar IN, Stanley SE: Systematic analysis of DNA microarray data: ordering and interpreting patterns of gene expression. Genome Res 2001, 11(7): 1149-1155.

2. Lee HK, Hsu AK, Sajdak J, Qin J, Pavlidis P: Coexpression analysis of human genes across many microarray data sets. Genome Res 2004, 14(6): 1085-1094.

3. Robertson $G$, Hirst $M$, Bainbridge $M$, Bilenky $M$, Zhao $Y$, Zeng $T$, Euskirchen G, Bernier B, Varhol R, Delaney A, et al: Genome-wide profiles of STAT1 DNA association using chromatin immunoprecipitation and massively parallel sequencing. Nat Methods 2007, 4(8): 651-657.

4. Mikkelsen TS, Ku M, Jaffe DB, Issac B, Lieberman E, Giannoukos G, Alvarez P, Brockman W, Kim TK, Koche RP, et al: Genome-wide maps of chromatin state in pluripotent and lineage-committed cells. Nature 2007, 448(7153): 553-560.

5. Marson A, Levine SS, Cole MF, Frampton GM, Brambrink T, Johnstone $S$, Guenther MG, Johnston WK, Wernig M, Newman J, et al: Connecting microRNA genes to the core transcriptional regulatory circuitry of embryonic stem cells. Cell 2008, 134(3): 521-533.

6. Barski A, Cuddapah S, Cui K, Roh TY, Schones DE, Wang Z, Wei G, Chepelev I, Zhao K: High-resolution profiling of histone methylations in the human genome. Cell 2007, 129(4): 823-837.

7. Yang MQ, Athey BD, Arabnia HR, Sung AH, Liu Q, Yang JY, Mao J, Deng Y: High-throughput next-generation sequencing technologies foster new cutting-edge computing techniques in bioinformatics. BMC genomics 2009, 10(Suppl 1): 11.

8. Lefrancois P, Euskirchen GM, Auerbach RK, Rozowsky J, Gibson T, Yellman CM, Gerstein M, Snyder M: Efficient yeast ChIP-Seq using multiplex short-read DNA sequencing. BMC genomics 2009, 10: 37.

9. Taylor J, Schenck I, Blankenberg D, Nekrutenko A: Using galaxy to perform large-scale interactive data analyses. In Current protocols in bioinformatics/ editoral board Edited by: Andreas D Baxevanis, et al 2007, Chapter 10(Unit 10): 15 .

10. Albert I, Wachi S, Jiang C, Pugh BF: GeneTrack-a genomic data processing and visualization framework. Bioinformatics 2008, 24(10): 1305-1306.
11. Ji H, Jiang H, Ma W, Johnson DS, Myers RM, Wong WH: An integrated software system for analyzing ChIP-chip and ChIP-seq data. Nat Biotechnol 2008, 26(11): 1293-1300.

12. Rozowsky J, Euskirchen G, Auerbach RK, Zhang ZD, Gibson T, Bjornson R, Carriero N, Snyder M, Gerstein MB: PeakSeq enables systematic scoring of ChIP-seq experiments relative to controls. Nat Biotechnol 2009, 27(1): 66-75.

13. Zhang $Y$, Liu T, Meyer CA, Eeckhoute J, Johnson DS, Bernstein BE, Nussbaum C, Myers RM, Brown M, Li W, et al: Model-based analysis of ChIP-Seq (MACS). Genome Biol 2008, 9(9): R137.

14. Gamage J, Weerahandi S: Size performance of some tests in one-way anova. Communications in Statistics - Simulation and Computation 1998, 27(3): 625-640.

15. Wittkowski KM: Statistical analysis of unbalanced and incomplete designs - experiences with BMDP and SAS. Statistical software newsletter 1991.

16. Qian F, Kruse U, Lichter P, Sippel AE: Chromosomal localization of the four genes (NFIA, B, C, and X) for the human transcription factor nuclear factor I by FISH. Genomics 1995, 28(1): 66-73.

17. Gronostajski RM: Roles of the NFI/CTF gene family in transcription and development. Gene 2000, 249(1-2): 31-45.

18. Santoro C, Mermod N, Andrews PC, Tjian R: A family of human CCAATbox-binding proteins active in transcription and DNA replication: cloning and expression of multiple cDNAs. Nature 1988, 334(6179): 218-224.

19. Roulet $E$, Bucher $P$, Schneider R, Wingender E, Dusserre $Y$, Werner $T$, Mermod N: Experimental analysis and computer prediction of CTF/NFI transcription factor DNA binding sites. J Mol Biol 2000, 297(4): 833-848.

20. Furlong EE, Rein T, Martin F: YY1 and NF1 both activate the human p53 promoter by alternatively binding to a composite element, and YY1 and E1A cooperate to amplify p53 promoter activity. Mol Cell Biol 1996, 16(10): 5933-5945.

21. Johansson EM, Kannius-Janson M, Bjursell G, Nilsson J: The p53 tumor suppressor gene is regulated in vivo by nuclear factor $1-C 2$ in the mouse mammary gland during pregnancy. Oncogene 2003, 22(38): 6061-6070.

22. Wickenheisser JK, Nelson-DeGrave VL, Quinn PG, McAllister JM: Increased cytochrome P450 17alpha-hydroxylase promoter function in theca cells isolated from patients with polycystic ovary syndrome involves nuclear factor-1. Mol Endocrinol 2004, 18(3): 588-605.

23. Ouellet S, Vigneault F, Lessard M, Leclerc S, Drouin R, Guerin SL: Transcriptional regulation of the cyclin-dependent kinase inhibitor $1 \mathrm{~A}$ (p21) gene by NFI in proliferating human cells. Nucleic Acids Res 2006, 34(22): 6472-6487.

24. Gao S, Zhao Y, Kong L, Toselli P, Chou IN, Stone P, Li W: Cloning and characterization of the rat lysyl oxidase gene promoter: identification of core promoter elements and functional nuclear factor I-binding sites. $J$ Biol Chem 2007, 282(35): 25322-25337.

25. O'Donnell A, Yang SH, Sharrocks AD: MAP kinase-mediated c-fos regulation relies on a histone acetylation relay switch. Mol Cell 2008, 29(6): 780-785.

26. Esnault G, Majocchi S, Martinet D, Besuchet-Schmutz N, Beckmann JS, Mermod N: Transcription factor CTF1 acts as a chromatin domain boundary that shields human telomeric genes from silencing. Mol Cell Biol 2009, 29(9): 2409-2418.

27. Ferrari S, Simmen KC, Dusserre Y, Muller K, Fourel G, Gilson E, Mermod N: Chromatin domain boundaries delimited by a histone-binding protein in yeast. J Biol Chem 2004, 279(53): 55520-55530.

28. Roulet E, Busso S, Camargo AA, Simpson AJ, Mermod N, Bucher P: Highthroughput SELEX SAGE method for quantitative modeling of transcription-factor binding sites. Nat Biotechnol 2002, 20(8): 831-835.

29. Plasari G, Calabrese A, Dusserre Y, Gronostajski RM, McNair A, Michalik L, Mermod N: Nuclear factor I-C links platelet-derived growth factor and transforming growth factor beta1 signaling to skin wound healing progression. Mol Cell Biol 2009, 29(22): 6006-6017.

30. Steele-Perkins G, Butz KG, Lyons GE, Zeichner-David M, Kim HJ, Cho MI, Gronostajski RM: Essential role for NFI-C/CTF transcription-replication factor in tooth root development. Mol Cell Biol 2003, 23(3): 1075-1084

31. Hebbar PB, Archer TK: Chromatin-dependent cooperativity between sitespecific transcription factors in vivo. J Biol Chem 2007, 282(11): 8284-8291.

32. Satoh S, Noaki T, Ishigure T, Osada S, Imagawa M, Miura N, Yamada K, Noguchi T: Nuclear factor 1 family members interact with hepatocyte 
nuclear factor 1alpha to synergistically activate L-type pyruvate kinase gene transcription. J Biol Chem 2005, 280(48): 39827-39834.

33. Zhao $L H, B a X Q$, Wang $X G$, Zhu $X J$, Wang $L$, Zeng $X L$ : BAF complex is closely related to and interacts with NF1/CTF and RNA polymerase II in gene transcriptional activation. Acta Biochim Biophys Sin (Shanghai) 2005, 37(7): 440-446.

34. Hebbar PB, Archer TK: Nuclear factor 1 is required for both hormonedependent chromatin remodeling and transcriptional activation of the mouse mammary tumor virus promoter. Mol Cell Biol 2003, 23(3): 887-898

35. Kannius-Janson M, Johansson EM, Bjursell G, Nilsson J: Nuclear factor 1-C2 contributes to the tissue-specific activation of a milk protein gene in the differentiating mammary gland. J Biol Chem 2002, 277(20): 17589-17596.

36. Ling G, Hauer CR, Gronostajski RM, Pentecost BT, Ding X: Transcriptional regulation of rat CYP2A3 by nuclear factor 1: identification of a novel $\mathrm{NFI-A}$ isoform, and evidence for tissue-selective interaction of NFI with the CYP2A3 promoter in vivo. J Biol Chem 2004, 279(27): 27888-27895.

37. Rossi P, Karsenty G, Roberts AB, Roche NS, Sporn MB, de Crombrugghe B: A nuclear factor 1 binding site mediates the transcriptional activation of a type I collagen promoter by transforming growth factor-beta. Cell 1988, 52(3): 405-414.

38. Fourel G, Boscheron C, Revardel E, Lebrun E, Hu YF, Simmen KC, Muller K, Li R, Mermod N, Gilson E: An activation-independent role of transcription factors in insulator function. EMBO Rep 2001, 2(2): 124-132.

39. Mikkelsen TS, Hanna J, Zhang X, Ku M, Wernig M, Schorderet P, Bernstein $B E$, Jaenisch R, Lander ES, Meissner A: Dissecting direct reprogramming through integrative genomic analysis. Nature 2008, 454(7200): 49-55.

40. ChIP-Seq Analysis Server. [http://ccg.vital-it.ch/chipseq/].

41. ChIP-Seq Analysis tools - download repository. [https://sourceforge.net/ projects/chip-seq/]

42. Galaxy tools. [http://main.g2.bx.psu.edu/].

43. Giardine B, Riemer C, Hardison RC, Burhans R, Elnitski L, Shah P, Zhang Y, Blankenberg D, Albert I, Taylor J, et al: Galaxy: a platform for interactive large-scale genome analysis. Genome Res 2005, 15(10): 1451-1455.

doi:10.1186/1471-2164-12-181

Cite this article as: Pjanic et al:: Nuclear factor I revealed as family of promoter binding transcription activators. BMC Genomics 2011 12:181.

\section{Submit your next manuscript to BioMed Central and take full advantage of:}

- Convenient online submission

- Thorough peer review

- No space constraints or color figure charges

- Immediate publication on acceptance

- Inclusion in PubMed, CAS, Scopus and Google Scholar

- Research which is freely available for redistribution 